\section{Dopamine and schizophrenia}

SIR - The authors of the papers on the cloning of the $\mathrm{D}$, dopamine receptor that recently appeared in Nature ${ }^{1-3}$ suggested that alterations in the number or activity of opposing $\mathrm{D}_{1}$ and $\mathrm{D}_{2}$ receptors may be a contributory factor in Parkinson's disease and schizophrenia in that $D_{1}$ receptors stimulate and $D_{2}$ receptors inhibit adenylyl cyclase. Seeman et al. ${ }^{+}$have demonstrated a link between $\mathrm{D}_{1}$ and $\mathrm{D}_{2}$ receptors located on the same postsynaptic membrane, showing that binding of a $\mathrm{D}_{2}$ agonist to striatal tissues is affected by pretreatment with $D_{1}$-selective antagonist and vice versa, and suggesting cross talk between the receptors mediated by G-protein interactions. This link is missing in more than half the postmortem striata from brains of people with schizophrenia and Huntington disease but is present in control striata ${ }^{+}$, indicating a molecular mechanism for the disease state.

The model is simple and attractive. It can be extended because the existence of separate stimulatory and inhibitory receptors for the same agonist in the same membrane has predictable consequences on cyclic AMP formation. I recently reported $^{5,6}$ a description of prostaglandinregulated cyclic AMP metabolism in blood platelets based on this model. I suggested that a distinct inhibitory receptor represents a general mechanism of

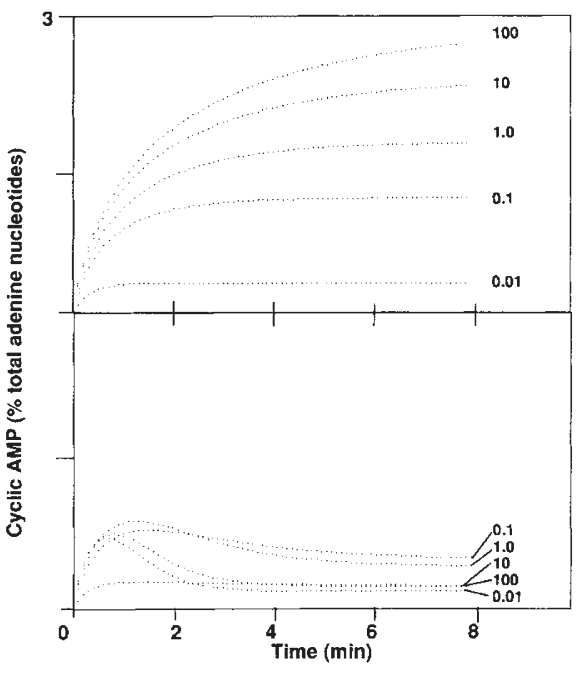

Computer simulations of steady-state cyclic AMP levels in intact cells based on actual data obtained for prostaglandin regulation of cyclic AMP metabolism in blood platelets ${ }^{5,6}$. Top, The steady-state level of cyclic AMP in the absence of an inhibitory receptor is determined by receptor-linked adenylyl cyclase activity, which increases as a saturable function of agonist concentration (numbers on right) and phosphodiesterase activity which is constant. Bottom, The inhibitory receptor, which con verts adenylyl cyclase to an inactive form, binds agonist more weakly than the stimulatory receptor so the concentration dependence of inhibition differs from that for stimulation. autacoid (or neurotransmitter) regulation, buffering cellular responses against the transient localized increases in agonist concentration that occur when agonists are produced close to their sites of action ${ }^{h}$. The figure shows a computer simulation of a dose-dependent rise in cyclic AMP with (bottom) and without (top) a slowacting inhibitory receptor that binds agonist more weakly than the stimulatory receptor.

I propose that $D_{1}$ and $D_{2}$ receptors together regulate adenylyl cyclase activity on the postsynaptic membrane so that cyclic AMP in neurons of normal individuals is maintained at an essentially constant level despite sharp fluctuations in agonist concentration that must occur when neurotransmitter is released in to the tiny volume of the synaptic cleft. Interference with this protective buffering mechanism, which may occur through a reduction or absence of the inhibitory receptor, would result in wild swings in the level of cyclic AMP, reflecting transient but physiological changes in neurotransmitter concentration. The effect of loss of control of dopamine-regulated cyclic AMP level may affect neuronal firing, perhaps explaining manifestations such as those observed in people with schizophrenia.

Department of Pharmacology, BARRIE ASHBY Temple University School of Medicine, Philadelphia,

Pennsylvania 19140, USA

1. Deary, A. et al. Nature 347, 72-76 (1990).

2. Zhou, Q.-Y. et al. Nature 347, 76-80 (1990)

Sunahara, R.K. et al. Nature 347, 80-83 (1990)

4. Seeman, P., Niznik, H.B., Guan, H.-C., Booth, G. \& Ulpian,

C. Proc. natn. acad. Sci. U.S.A. 86, 10156 (1989)

. Ashby, B. Molec. Pharmac. 36, 866-873 (1989).

6. Ashby, B. Molec. Pharmac. 38. 46-53 (1990).

\section{Red herring}

SIR - I suspect that the claim by Fry and Bonnet-Bidaud (Nature 347, 625; 1990) that an entry in the Han dynasty record book has a bearing on the putative ancient red colour of Sirius is a red herring. The textual statement "At East there is big star called Wolf-horn changes colour" has a sense of immediacy of the sort probably arising from noticing that Sirius was chromatically scintillating with unusual violence as it rose in the east. This relatively unremarkable observation could well produce the commonplace and secure prophecy of "many thiefs, robbers". A major and permanent change in colour of the brightest star in the sky would be more likely to imbue the astrologer with the courage to predict failures of harvests or falls of emperors.

BRIAN WARNER

Department of Astronomy,

University of Texas,

Austin,

Texas 78712, USA

\section{Equal animals}

SIR-May in News and Views ${ }^{1}$ discusses the question of whether all species should be equally valued from the perspective of conservation biology. $\mathrm{He}$ and others ${ }^{2.3}$ have concluded that some animals must be considered more equal than others ${ }^{4}$ when assigning spaces in a shrinking - if not sinking - ark.

But how can one assess the relative worth of, for example, two different species of lemur? If diversity is the goal, the key seems to lie in taxonomy, with species for which no close relatives exist receiving greater weight. May described several weighting schemes based on the branching order, or topology, of an evolutionary tree relating the species in question. The length of the branches of such a tree, corresponding to degrees of change between evolutionary diversions, should also be taken into account. The two evolutionary trees shown in the figure,

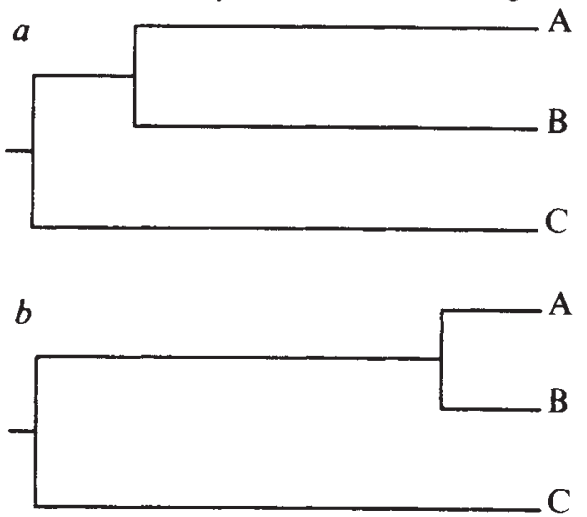

for example, are topologically identical. Yet the three species in tree $a$, having diverged at nearly the same time, should receive roughly equal weights, while in tree $b$, species $\mathrm{C}$ should count almost as much as species A and B combined.

This intuition was first given rigorous treatment by Felsenstein ${ }^{5.6}$ in the context of phylogenetic reconstruction. More recently, we have argued ${ }^{7}$ that Felsenstein's formulation has general applicability to the problem of assessing correlated data related by an evolutionary tree. Conservation biology appears to be an additional field to which these ideas apply.

Felsenstein's weights can be summarized by imagining an evolutionary tree as being constructed of resistant wire. If each leaf is 'grounded', and one volt is applied to the node representing the common ancestor, then the current flowing out of a leaf of the tree represents the relative contribution the corresponding species makes to the total diversity ${ }^{5-?}$.

These weights are meaningful only in the context of the complete tree, and change if any leaves are pruned. One cannot assign to the various species fixed values which can be added to assess a subcollection. Instead, if one must choose among several subtrees, the one with the 